\title{
Standardized Bowel Training Benefits Patients with Neurogenic Bowel Dysfunction After Spinal Cord Injury
}

\author{
Cuiqing Liu*, Lihuan Hou, Hong Zhang \\ The First Affiliated Hospital of Jinan University, Guangzhou, China
}

Email address:

cuiqing.liu@yahoo.com (Cuiqing Liu)

To cite this article:

Cuiqing Liu, Lihuan Hou, Hong Zhang. Standardized Bowel Training Benefits Patients with Neurogenic Bowel Dysfunction After Spinal Cord Injury. American Journal of Nursing Science. Vol. 4, No. 6, 2015, pp. 305-307. doi: 10.11648/j.ajns.20150406.12

\begin{abstract}
In order to evaluate the effect of standardized management on spinal cord injury patients with neurogenic bowel dysfunction, 50 cases of spinal cord injury patients from January 2011 to December 2013 were treated with neurogenic bowel intestinal standardized management. The effect was compared to that with routine nursing of 32 spinal cord injury patients from January 2009 to December 2010. Data analysis regarding the age, spinal cord injury, neurological movement and basic sensory score showed no statistically significant difference between the two groups $(\mathrm{P}>0.05)$. However, bowel functional indexes including abdominal distension, abdominal pain, evacuation time and anal incontinence of the standardized training group decreased as compared to those of control group $(\mathrm{P}<0.05)$. The bowel way of the standardized training patients was also in line with normal living habits, including fixed time and the toilet bowel movement. In conclusion, standardization bowel management intervention is beneficial to the patients with spinal cord injury to form regular bowel habit.
\end{abstract}

Keywords: Spinal Cord Injury, Neurogenic Bowel Dysfunction, Standardized Bowel Training

\section{Introduction}

Neurogenic bowel dysfunction (NBD) caused by spinal cord injury (SCI) would lead to intestinal sensorimotor disorders. The consequences include altered bowel motility, loss of sphincter control and an inability significantly to increase intra-abdominal pressure $[1,2]$. Bowel care is a time-consuming work, leading to an increase in the level of anxiety and depression [3-5]. An effective bowel program should cover the issues of constipation, effective evacuation and prevention of complications $[1,6-8]$. The objective of the current study was to evaluate the improved bowel management applied to 50 individuals with SCI in hospital from Year 2011 to 2013, when comparing with 32 individuals by normal care from Year 2009 to 2010.

\section{Materials and Methods}

\subsection{Subject Selection}

Fifty patients with SCI from Jan, 2011 to Dec, 2013 in the First Affiliated Hospital of Jinan University were selected as treatment group, containing 36 men and 14 women with average age $30 \pm 10.2$. Injury type: 20 cases of complete injury; 30 cases of non-complete injury; 44 cases with thoracic lumbosacral injury; 6 cases of cervical injury. Inclusion criteria: after spinal shock, no original intestinal disease; patient or his/her caregiver willing to cooperate. Thirty-two SCI patients with bowel dysfunction during Jan, 2009 to Dec, 2010 with routine nursing were selected into control group by a retrospective analysis.

\subsection{Standardized Bowel Training}

Fifty individuals in treatment group underwent standardized bowel management. Intestinal stool was cleared by enema or oral laxatives 3 days before the training. The standardized bowel training process was as follows. Step 1, develop a diet plan to make sure an amount of intake of water and cellulose. Step 2, guide the patients to massage the lower abdomen clockwise for $20 \mathrm{~min}$, from ascending colon, transverse colon and descending colon to sigmoid; with light to heavy pressure, then to light pressure. Guide the patients' external anal sphincter to close and levator ani muscle to increase the nerve sensitivity. Step 3, stimulate the skin around the anus, with an interval of $2 \mathrm{~min}$. Step 4, simple evacuation: $40 \mathrm{~mL}$ glycerine enema insertion into rectal ampulla or deeper parts. Step 5, tell patients not to evacuate with heavy pressure first; 10 min after the management, massage then try evacuate; or manual evacuation. 
Standardized bowel training was taken every day or every other day with a fixed time, based on the psychological need, bowel habit before injury and injury severity. The amount of water intake in Step 1 was combined with bladder training. Step 2 was taken every day. Step 3 was decided by the tightness of the anus, if it did not work then performed Steps 4 and 5. A table was developed to record the bowel situation; the training was last for 2 weeks to observe the best bowel method for each patient, then other methods were taught.

Patients in control group were nursed by routine care, including cleaning care to independent evacuation patients; laxatives given and dietary guidance when constipation happened; skin care and antidiarrheal treatment when diarrhea happened.

\subsection{Observing Index}

The evaluation of bowel recovery of neurogenic bowel dysfunction has been started since last century and bowel dysfunction and the associated psychological abnormalities were evaluated by intestinal function and survival quantity [9]. In China, the measurement method of intestinal function is always taken by determination of colonic transit time [10] and anorectal manometry $[11,12]$. But during the actual nursing practice, these indexes are hard to achieve. So we took some effective and measurable observation indexes including 1) with/without bloating or abdominal pain; 2) independent evacuation, fixed evacuation every day or every other day by rectal irritation or laxatives, with soft defecate; 3) no bowel incontinence between evacuations; 4) the times of evacuation less than $30 \mathrm{~min}$.

\subsection{Statistical Analysis}

Age, sense and movement or other indexes between the treated group and control group were analyzed by SPSS 19.0. The statistical significance of the differences was analyzed using Student's $t$-test between two groups. $P<0.05$ was considered to be statistically significant.

\section{Results}

According to the American Spinal Injury Association (ASIA) [13], age, moter, sensory scores were evaluated between the standardized group and control group. Statistical data were presented as mean \pm SD. $P>0.05$ was considered as significant difference. The results shown in Table 1 indicated that the SCI severity between the standardized group and control group had no significant differences.

Table 1. ASIA scores between the standardized and control groups (mean $\pm S D$ ).

\begin{tabular}{|c|c|c|c|c|c|}
\hline \multirow{2}{*}{ Group } & \multirow{2}{*}{ Cases (N) } & \multirow{2}{*}{ Age } & \multirow{2}{*}{ Moter score } & \multicolumn{2}{|l|}{ Sensory score } \\
\hline & & & & Light Touch & Pin Prick \\
\hline Standardized group & 50 & $33.03 \pm 10.78$ & $53.76 \pm 22.08$ & $72.48 \pm 19.67$ & $72.93 \pm 20.91$ \\
\hline Control group & 32 & $29.68 \pm 9.66$ & $49.86 \pm 21.18$ & $70.41 \pm 20.21$ & $70.55 \pm 23.13$ \\
\hline$F$ value & & 0.965 & 0.344 & 0.049 & 0.557 \\
\hline$P$ value & & 0.331 & 0.560 & 0.826 & 0.459 \\
\hline
\end{tabular}

Evaluation of intestinal function was performed as mentioned in the method section. The percentage scores were shown in Table 2. These data showed that the standardized bowel training significantly reduced the bloating or abdominal pain, increased independent evacuation by patients themselves, reduced bowel incontinence and shortened the time of evacuation.

Table 2. Evaluation of intestinal function between the standardized and control groups.

\begin{tabular}{|c|c|c|c|c|}
\hline & Standardized group $(\mathrm{N}=50)$ & Control group $(\mathrm{N}=32)$ & $\mathrm{Z}$ index & P value \\
\hline Bloating or abdominal pain $[\mathrm{n}(\%)]$ & $0(0 \%)$ & $19(59 \%)$ & -6.178 & 0.00 \\
\hline Fixed independent evacuation $[\mathrm{n}(\%)]$ & $50(100 \%)$ & $10(31 \%)$ & -7.024 & 0.00 \\
\hline Incontinence $[\mathrm{n}(\%)]$ & $3(6 \%)$ & $21(66 \%)$ & -5.753 & 0.00 \\
\hline Evacuation less than $30 \min [\mathrm{n}(\%)]$ & $40(80 \%)$ & $8(25 \%)$ & -4.901 & 0.00 \\
\hline
\end{tabular}

\section{Discussion}

The current study revealed that after bowel management, intestinal functions including bloating, abdominal pain, fixed independent evacuation, incontinence and evacuation time of the patients with SCI were largely relieved or shortened, as compared to control group with regular nursing care. The evacuation way (referred to fixed evacuation time in toilet) was close to normal life, indicating the successful achievement of standardized bowel training of SCI patients.

Neurogenic bowel dysfunction (NBD) can be divided into reflexic and areflexic types. Because the undamaged spinal cord (S2-S4), fulfilled stool can stimulate the evacuation of patients with reflexic NBD, but the process is not controlled by brain, leading to incontinence and incomplete evacuation; in patients with areflexic NBD, these stimulation wouldn't cause evacuation. Therefore, during the early period of bowel training, we often apply enema or laxatives to empty the intestines, thus reducing constipation and incontinence.

The opportunity is large for incomplete paraplegia patients with functional external anal sphincter to recover spontaneous evacuation. Patients with SCI, especially those with unaffected arms and hands could be reemployed and get back to work. Factors that affect the reemployment are multiple. SCI results in the loss of normal motor and sensory function, 
leading to severe disability and reduced quality of life [14]. A research showed that $1 / 3$ of SCI patients thought that after SCI stabilized, the impact of intestinal dysfunction was more severe than that of bladder or sex dysfunction, and $41 \%$ thought that bowel dysfunction was a moderate or severe problem that affects lifespan [15]. Bowel dysfunction would lead to a series of problems such as restricted outdoor activity, mental stress and other psychological pressure. Obviously, the early intervention with standardized bowel training would largely contribute to the forming of ordered and regular evacuation of SCI patients, further reducing their mental stress, increasing the reemployment desire and finally benefiting the life quality of these SCI patients.

\section{Conclusion}

Neurogenic bowel dysfunction is important for the quantified life in persons with spinal cord injury, especially in physical functioning and physical component summary. Standardization bowel management intervention benefits the patients with spinal cord injury to form regular bowel habit, thus improving the life living quantity.

\section{Acknowledgement}

The authors declare no conflict of interest. This work was supported by Medical Scientific Research Foundation of Guangdong Province, China (No. A2012344).

\section{References}

[1] Lynch, A.C., et al., Bowel dysfunction following spinal cord injury. Spinal Cord, 2001. 39(4): p. 193-203.

[2] Noonan, V.K., et al., Impact of associated conditions resulting from spinal cord injury on health status and quality of life in people with traumatic central cord syndrome. Arch Phys Med Rehabil, 2008. 89(6): p. 1074-82.

[3] Glickman, S. and M.A. Kamm, Bowel dysfunction in spinal-cord-injury patients. Lancet, 1996. 347(9016): p. 1651-3.
[4] Kirshblum, S.C., et al., Bowel care practices in chronic spinal cord injury patients. Arch Phys Med Rehabil, 1998. 79(1): p. 20-3.

[5] Lynch, A.C., et al., Bowel dysfunction following spinal cord injury: a description of bowel function in a spinal cord-injured population and comparison with age and gender matched controls. Spinal Cord, 2000. 38(12): p. 717-23.

[6] Harari, D., et al., Constipation-related symptoms and bowel program concerning individuals with spinal cord injury. Spinal Cord, 1997. 35(6): p. 394-401.

[7] Coggrave, M.J. and C. Norton, The need for manual evacuation and oral laxatives in the management of neurogenic bowel dysfunction after spinal cord injury: a randomized controlled trial of a stepwise protocol. Spinal Cord, 2010. 48(6): p. 504-10.

[8] Stiens, S.A., S.B. Bergman, and L.L. Goetz, Neurogenic bowel dysfunction after spinal cord injury: clinical evaluation and rehabilitative management. Arch Phys Med Rehabil, 1997. 78(3 Suppl): p. S86-102.

[9] Kennedy, P., et al., Psychological contributions to functional independence: a longitudinal investigation of spinal cord injury rehabilitation. Arch Phys Med Rehabil, 2011. 92(4): p. 597-602.

[10] Fynne, L., et al., Gastric and small intestinal dysfunction in spinal cord injury patients. Acta Neurol Scand, 2012. 125(2): p. 123-8.

[11] Tornblom, H., et al., Colonic transit time and IBS symptoms: what's the link? Am J Gastroenterol, 2012, 107(5): p. 754-60.

[12] Lynch, A.C., et al., Anorectal physiology following spinal cord injury. Spinal Cord, 2000. 38(10): p. 573-80.

[13] Committee, M., et al., International standards for neurological classification of spinal cord injury, revised 2011. Top Spinal Cord Inj Rehabil, 2012. 18(1): p. 85-99.

[14] Guo, J.D., et al., Genetic ablation of receptor for advanced glycation end products promotes functional recovery in mouse model of spinal cord injury. Mol Cell Biochem, 2014. 390(1-2): p. $215-23$.

[15] Liu, C.W., et al., Relationship between neurogenic bowel dysfunction and health-related quality of life in persons with spinal cord injury. J Rehabil Med, 2009. 41(1): p. 35-40. 